\title{
Availability estimation of wind power forecasting and optimization of day-ahead unit commitment
}

\author{
Yun TENG ${ }^{1}$, , Qian HUI ${ }^{1,2}$, Yan $\mathrm{LI}^{3}$, Ouyang $\mathrm{LENG}^{4}$, \\ Zhe CHEN ${ }^{1,5}$
}

\begin{abstract}
Due to the uncertainty of the accuracy of wind power forecasting, wind turbines cannot be accurately equated with dispatchable units in the preparation of a dayahead dispatching plan for power grid. A robust optimization model for the uncertainty of wind power forecasting with a given confidence level is established. Based on the forecasting value of wind power and the divergence function of forecasting error, a robust evaluation method
\end{abstract}

CrossCheck date: 28 August 2019

Received: 21 November 2018/ Accepted: 28 August 2019/Published online: 16 November 2019

(C) The Author(s) 2019

$\triangle$ Yun TENG

tengyun@sut.edu.cn

Qian HUI

bofudeng58@163.com

Yan LI

sygdty@163.com

Ouyang LENG

360264936@qq.com

Zhe CHEN

zch@et.aau.dk

1 Shenyang University of Technology, Shenyang 110870 , China

2 State Grid Liaoning Electric Power Research Institute Customer Service Center, Shenyang 110004, China

3 State Grid East Inner Mongolia Electric Power Co, Ltd., Huhhot 010020, China

4 Economic and Technological Research Institute of State Grid East Inner Mongolia Electric Power Co. Ltd, Huhhot 010020, China

5 Aalborg University, Aalborg, Denmark for the availability of wind power forecasting during given load peaks is established. A simulation example is established based on a power system in Northeast China and an IEEE 39-node model. The availability estimation parameters are used to calculate the equivalent value of wind power of the conventional unit to participate in the dayahead dispatching plan. The simulation results show that the model can effectively handle the challenge of uncertainty of wind power forecasting, and enhance the consumption of wind power for the power system.

Keywords Consumption of wind power, Wind power availability, Uncertainty of wind power forecasting, Coordination dispatching, Robust estimation

\section{Introduction}

With the ongoing global petrochemical energy depletion, environmental degradation is an increasingly serious situation. The global Energy Internet, with renewable energy, has emerged as the main energy supply form and electric energy as the main energy carrier, which is an inevitable trend for the development of human energy system and energy industry [1-3]. In addition, such power generations with renewable energy such as wind power and photovoltaic power, have transformed the pattern of modern power system and the form of power generation [4]. In the current pattern, one of the serious challenges is that the operation of power grid requires stability, while the integration scale of fluctuating unpredicted renewable energies has been increasing $[5,6]$.

Wind power generation is now a key component in renewable energy generation. In 2016, the installed capacity in China increased to 169 billion $\mathrm{kW}$ while the 
abandoned wind power increased to 49.7 billion $\mathrm{kW}$, a $50 \%$ increase over the previous year, with $17 \%$ rate of curtailed power [7-9]. The main reason is that the output of wind power is obviously random and uncertain due to the climate and environment change. The increasing scale of the existing system, including wind power, will have to guarantee a stronger power balance in power system and make its operation less hazardous. Therefore, the current methods are being challenged [10-13].

Wind power forecasting and its ongoing improvements are eye-catching in wind power industries and the management of power system. So far, the studies of wind power forecasting and its improvement are based on the assumption that completely accurate information of geographic environment and climate is given. Then, the investigation of theory or method about the relationship between this information and wind power capacity is determined. And on this basis, the probability distribution of errors is forecasted [14, 15]. At present, the general method within and outside China is based on the criteria of climate, namely, the improvement of forecasting accuracy of wind power output. The model of the numerical weather prediction (NWP) as well as the information about the developer, is listed in [16]. On such a basis, the Kalman filtering algorithm is proposed in [17] to filter the output of the SKIRON NWP model, which significantly improves the forecasting accuracy of wind speed and wind power.

A scenario tree tool is developed in [17], which allows the statistics of wind power forecasting error to be altered and facilitates the study of how these statistics impact unit commitment and system operation. A general methodology for deriving optimal bidding strategies based on probabilistic forecasting of wind generation is proposed in [18], and the sensitivity of wind power costs has been analyzed. The opportunities available are explored for wind power producers (WPPs) if they can purchase or schedule some reserves to offset part of their deviations rather than being fully penalized in the real-time market, and the revenue for WPPs with such mechanisms is modeled in [19].

However, as the dispatching systems of power grid are facing more and more uncertainties related to wind power forecasting errors, the scheduling system of power grid has insufficient information to handle these uncertainties under the fast increasing scale of wind power integration/interconnection among various energies. Therefore, the current method demanding accurate information cannot fulfill the need in the real work of the scheduling system [20-23]. It is impossible to take the forecasting value of wind power as the effective power output in day-ahead dispatching plan based on the reliability of power supply during the peak load period. This results in a large number of curtailed wind power due to the minimum output limit of conventional units during a low-load period.
Therefore, wind power availability can be accurately evaluated or calculated by using a robust estimation. The minimum possible wind power output can be inferred based on the forecasting results of wind power, so that wind power can be used as a reliable source to replace a certain proportion of conventional energy units and participate in the day-ahead dispatching plan of power system. To improve the efficiency of unit commitment, it is necessary to reduce the number of unit start-ups and shutdowns, and to have a decisive role in significantly reducing the curtailed wind.

A robust estimation model for the maximum probability rate of wind power prediction and its probability distribution is proposed, which is different from the traditional theory of improvement of wind power forecasting accuracy. With the current theory of prediction of wind power, based on the results of wind power forecasting, the availability of wind power during the peak load period can be obtained. According to their availability, the unit commitment can be better arranged to tackle the conflict between the fluctuation of wind power and the reserve capacity, so the challenge of dispatching faced by the power grid with large-scale wind power can be handled.

\section{Availability index of wind power forecasting}

\subsection{Definition of accuracy of wind power forecasting}

Assume that the time series of the actual output of wind power is $x_{i}$, and the predicted counterpart is $x_{i}^{\prime}$. The absolute value of the error of the wind power forecasting at a future time can be expressed as:

$e_{i}=\left|x_{i}^{\prime}-x_{i}\right|$

This paper defines the accuracy of wind power forecasting at a future time as:

$R_{\mathrm{A}}=\frac{x_{i}^{\prime}-x_{i}}{x_{i}^{\prime}} \quad i=\left\{i \mid x_{i}<x_{i}^{\prime}\right\}$

where $R_{\mathrm{A}}$ is the accuracy of wind power forecasting.

\subsection{Definition of availability of wind power forecasting}

According to the conception of the accuracy of wind power forecasting, the availability index for wind power forecasting is defined. Different from the evaluation of conventional wind power forecasting error or the difference between the estimated value and the actual output, the availability index is able to reflect the minimum possible value of wind power during the peak load period, or the maximum possible value of wind power forecasting error, 
and the possibility of the lowest wind power output based on the forecasting results.

The definition of the availability index of wind power forecasting is:

$\left\{\begin{array}{l}Q=\max \left(\frac{P_{W A}+P_{W S}}{P_{W F}}\right) \\ P_{W I}=Q \cdot P_{W F}\end{array}\right.$

where $Q$ is the day-ahead availability of wind power forecasting; $P_{W A}$ is the actual wind power during the peak load period; $P_{W S}$ is the actual curtailed wind power; $P_{W F}$ is the estimated value of wind power during the day-ahead peak load period; and $P_{W I}$ is the capacity of the output of virtual wind power unit participating in the day-ahead scheduling plan during predicted peak load period.

In the optimum unit commitment of the day-ahead dispatching, according to the availability $Q$, the value of the wind power forecasting is equivalent to the virtual unit to participate in the unit commitment optimization, which can effectively reduce the number of running machines.

\section{Robust estimation model of availability of wind power forecasting}

\subsection{Robust estimation of model during various peak load periods}

During a day-ahead dispatching period, the power grid faces a scenario $d$ with uncertain wind power forecasting, so the arrangement of the day-ahead scheduling plan requires the availability $Q$ and its probability $c$ given by wind power enterprises. In the actual scenario, the output given by the enterprise is $r$. After a dispatching period, if the actual output of wind power is less than the corresponding scale of availability, the power of other power sources will be $P_{s}$. If the actual output of wind power is more than the corresponding scale of credibility, the surplus will be the amount of curtailed wind power $v$.

Therefore, the issues of unit commitment of day-ahead dispatching will be equivalent to those of ensuring the optimum credibility $Q^{*}$ of wind power forecasting at the beginning of the day-ahead dispatching period. And then the product between $Q^{*}$ and the results of wind power forecasting is regarded as the maximum available power of a controllable source, which is the same as the virtual unit to participate in the day-ahead unit commitment plan.

With the load data, the value of wind power forecasting, the actual output of wind power without the availability of wind power forecasting and its statistical distribution, will be the forecasted scenario $d$ which contains $m$ number of scenarios, according to the duration of peak load period:

$d=\left\{d_{1}, d_{2}, \ldots, d_{m}\right\}$

The value of each scenario $d_{i}(i=1,2, \ldots, m)$ corresponds to one duration of the load peak. In scenario $d_{i}$, the probabilities of wind power forecasting error $e_{i}$ $(i=1,2, \ldots, m)$ are assumed to be:

$P_{r}\left(d=d_{i}\right)=p_{i}$

where $p_{i} \geq 0$ and $\sum_{i=1}^{m} p_{i}=1$.

In order to make the wind power forecasting credibility $Q$ accurately reflect the actual output of the wind power, the fitness function is defined as:

$$
\begin{aligned}
\zeta\left(Q ; d_{i}\right)= & r \min \left(d_{i}, Q\right)-P_{s} \max \left(d_{i}-Q\right) \\
& +\max \left(Q-d_{i}\right)-c Q
\end{aligned}
$$

The optimal value of availability of wind power forecasting is:

$Q^{*}=\arg \min \left\{E\left[\zeta\left(Q ; d_{i}\right)\right]=\sum_{i=1}^{m} p_{i} \zeta\left(Q ; d_{i}\right)\right\}$

where $E[\cdot]$ is the expectation operator.

If $\boldsymbol{p}=\left(p_{1}, p_{2}, \ldots, p_{m}\right)^{\mathrm{T}}$ is the undetermined probability vector of the error of wind power forecasting sometime in the future, then under the uncertain distribution of probability of wind power forecasting error, the robust estimation of availability of wind power forecasting can be expressed as:

$$
\begin{aligned}
& \max _{Q, \alpha_{Q}} \min _{\boldsymbol{p}}\left\{\alpha_{Q}-\frac{1}{\beta} E\left\{\max \left[\alpha_{Q}-\zeta\left(Q ; d_{i}\right)\right]\right\}\right\} \\
& =\max _{Q, \alpha_{Q}} \min _{\boldsymbol{p}}\left\{\alpha_{Q}-\frac{1}{\beta} \sum_{i=1}^{m} p_{i} \max \left[\alpha_{Q}-\zeta\left(Q ; d_{i}\right)\right]\right\}
\end{aligned}
$$

where $\beta$ is the fitness risk tolerance, which indicates that the wind power is less than the risk probability of the credible wind power output; and $\alpha_{Q}$ is the threshold of the fitness function $\xi(Q ; d)$.

By introducing the assistant vector $\boldsymbol{u}=\left(u_{1}, u_{2}, \ldots, u_{m}\right)^{\mathrm{T}}$, (5) is transformed into the robust optimization:

$$
\begin{cases}\max _{Q, \alpha_{Q}, \vartheta, \boldsymbol{u}} \vartheta \\ \text { s.t. } \quad \min _{\boldsymbol{p}} \alpha_{Q}-\frac{1}{\beta} \boldsymbol{p}^{\mathrm{T}} \boldsymbol{u} \geq \vartheta \\ & u_{i} \geq \alpha_{Q}-\zeta\left(Q ; d_{i}\right) \quad i=1,2, \ldots, m \\ & u_{i} \geq 0 \quad i=1,2, \ldots, m\end{cases}
$$

where $\left(Q, \alpha_{Q}, \vartheta, u\right) \in \mathrm{R} \times \mathrm{R} \times \mathrm{R} \times \mathrm{R}^{m}$; and $\vartheta$ is the auxiliary parameter. 


\subsection{Solution of robust estimation model}

Since (9) includes the undetermined probability vector $\boldsymbol{p}$, the direct solution is hardly known. In this paper, the above optimum is transformed into the estimation of the confidence region of wind power forecasting availability to fulfill the given confidence level. Therefore, the optimum issue of (9) is turned into the issue of robust planning.

Assume that the probability vector of wind power forecasting error during every peak load period is $\boldsymbol{p}=\left(p_{1}, p_{2}, \ldots\right.$, $\left.p_{m}\right)^{\mathrm{T}}$, where $p_{i}(i=1,2, \ldots, m) \geq 0$, and the probability vector of availability of wind power forecasting in the scenarios is $\boldsymbol{q}=\left(q_{1}, q_{2}, \ldots, q_{m}\right)^{\mathrm{T}}$, where $q_{i}(i=1,2, \ldots, m) \geq 0$. The divergence function between the forecasting availability and probability of forecasting error is $\phi$ [24], then:

$I_{\phi}(\boldsymbol{p}, \boldsymbol{q})=\sum_{i=1}^{m} q_{i} \phi\left(\frac{p_{i}}{q_{i}}\right)$

where $I_{\phi}(\boldsymbol{p}, \boldsymbol{q})$ is the divergence function of vectors $\boldsymbol{p}$ and $q$.

Suppose that the forecasted result samples are $N$, then under the confidence level of $1-K$ where $K$ is the significance level, the confidence region of the probability $\boldsymbol{p}$ of the wind power prediction availability under each scenario is:

$\mu_{N}=\left\{\boldsymbol{p} \in \mathrm{R}^{m} \mid p_{i} \geq 0, \boldsymbol{e}^{\mathrm{T}} \boldsymbol{p}=1, I_{\phi}\left(\boldsymbol{p}, \hat{\boldsymbol{p}}_{N}\right) \leq \rho\right\}$

where $\rho$ is auxiliary parameters; $\boldsymbol{e}^{\mathrm{T}}=\left(e_{1}, e_{2}, \ldots, e_{m}\right)$ is the error vector under every scenario; $\hat{\boldsymbol{p}}_{N}=$ $\left(p_{1, N}, p_{2, N} \ldots, p_{m, N}\right)$ is the maximum likelihood estimation of probability $\boldsymbol{p}$ based on $N$ samples of a small scale:

$\rho=\frac{\phi^{\prime \prime}(1)}{2 N}\left(\sqrt{\delta_{\phi}} \chi_{m-1,1-K}^{2}+\gamma_{\phi}\right)$

where $\chi_{m-1,1-K}^{2}$ is the value of the chi-square function of $m-1$ under the confidence interval of $1-K ; \delta_{\phi}$ and $\gamma_{\phi}$ are correction parameters defined in (13) and (14), respectively; and $\phi^{\prime \prime}, \phi^{(3)}$ and $\phi^{(4)}$ are the second, third and fourth derivatives of $\phi(t)=\frac{1}{t}(t-1)^{2}$, respectively.

$$
\begin{aligned}
\delta_{\phi}= & 1+\frac{1}{2(m-1) N}\left\{\left(2-2 m-m^{2}+s\right)\right. \\
& +\frac{2 \phi^{(3)}(1)}{\phi^{\prime \prime}(1)}\left(4-6 m-m^{2}+3 s\right) \\
& +\frac{1}{3}\left(\frac{\phi^{(3)}(1)}{\phi^{\prime \prime}(1)}\right)^{2}\left(4-6 m-m^{2}+5 s\right) \\
& \left.+\frac{2 \phi^{(4)}(1)}{\phi^{\prime \prime}(1)}(1-2 m+s)\right\}
\end{aligned}
$$

$$
\begin{aligned}
\gamma_{\phi}= & (m-1)\left(1-\sqrt{\delta_{\phi}}\right) \\
& +\frac{1}{N}\left(\frac{\phi^{(3)}(1)}{3 \phi^{\prime \prime}(1)}(2-3 m+s)+\frac{\phi^{(4)}(1)}{4 \phi^{\prime \prime}(1)}(1-2 m+s)\right)
\end{aligned}
$$

where $s$ is the auxiliary parameter and $s=\sum_{i=1}^{m} \frac{1}{p_{i, N}}$.

Then, under the uncertain probability set, the minimum constraint problem of equation (9) is turned into:

$\left\{\begin{array}{l}\alpha_{Q}-\eta-\lambda \rho-\lambda \sum_{i=1}^{m} p_{i, N} \phi^{*}\left(\frac{\mu_{i} / \beta-\eta}{\lambda}\right) \geq \vartheta \\ \lambda \geq 0\end{array}\right.$

where $\phi^{*}(\cdot)$ is the conjugate function of $\phi(\cdot) ; \eta$ and $\lambda$ are auxiliary parameters. The issue about parameter optimization in equation (6) can be turned into the one concerning robust planning:

$$
\begin{cases}\max _{Q, \vartheta, \lambda, \eta, \boldsymbol{u}} \vartheta \\ \text { s.t. } & \alpha_{Q}-\eta-\lambda \rho-\lambda \sum_{i=1}^{m} p_{i, N} \phi^{*}\left(\frac{\mu_{i} / \beta-\eta}{\lambda}\right) \geq \vartheta \\ & u_{i} \geq \alpha_{Q}-\zeta\left(Q ; d_{i}\right) \quad i=1,2, \ldots, m \\ & u_{i} \geq 0 \quad i=1,2, \ldots, m \\ & \lambda \geq 0\end{cases}
$$

where $(Q, \vartheta, \lambda, \mu, \boldsymbol{u}) \in \mathrm{R} \times \mathrm{R} \times \mathrm{R} \times \mathrm{R} \times \mathrm{R}^{m}$.

\section{Estimation of algorithm of wind power forecasting availability and application analysis}

\subsection{Robust estimation algorithm of wind forecasting availability}

Based on load data, wind power prediction data, actual wind power data and wind curtailment data in Northeast China from 2014 to 2016, a $\varphi$-divergence robust estimation model concerning the availability of wind power forecasting has been built. Based on wind power forecasting data used in day-ahead dispatching, the availability of wind power forecasting is estimated.

The typical daily load curves of the power grid of the region in winter and summer are shown in Fig. 1.

The time of the daily maximum load of the system and the distribution of errors of wind power forecasting are shown in Figs. 2 and 3, respectively.

According to the analysis, the number of scenarios of the peak load duration corresponding to wind power forecasting error is $m=10$. From the historical data analysis, the actual probability of wind power forecasting error is $p_{0}$ in each scenario of the regional power grid. Parameters related to the robust optimization model of availability of 


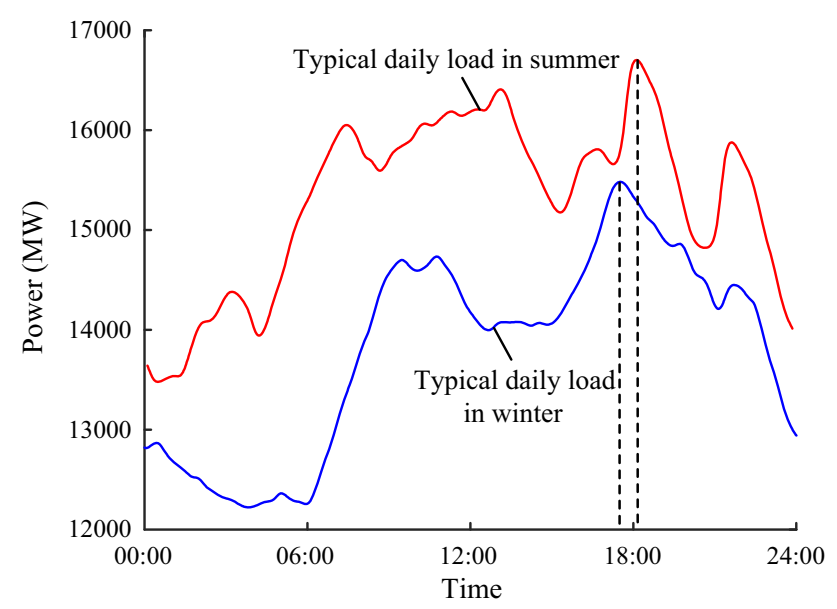

Fig. 1 Curves of typical daily load of system

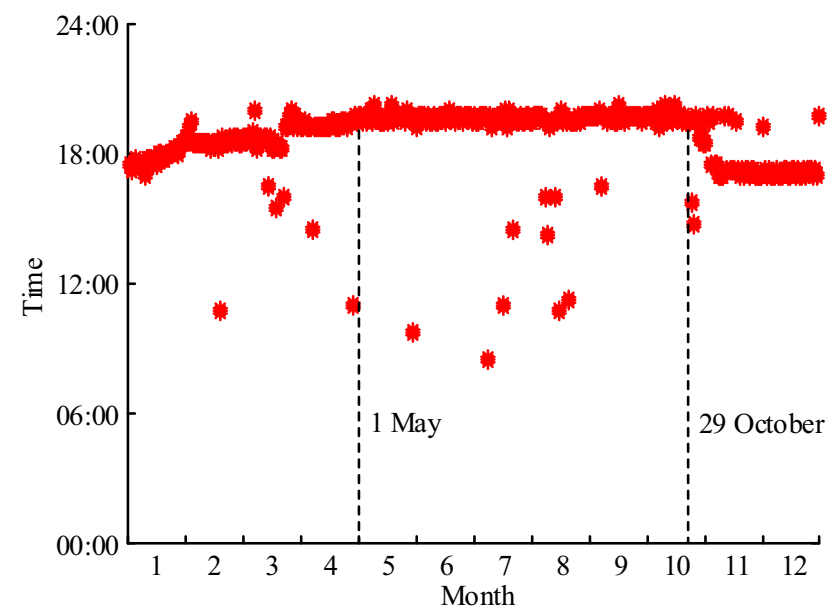

Fig. 2 Periods of daily maximum load of system

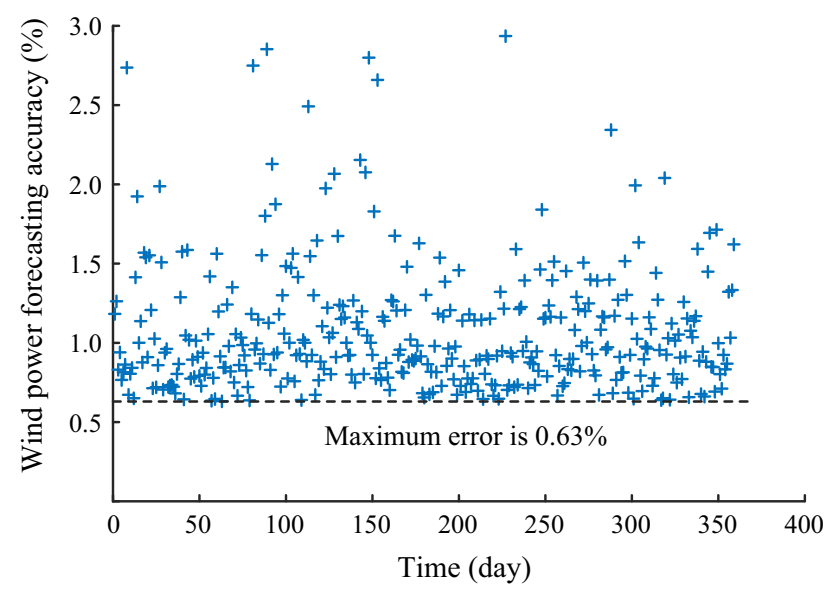

Fig. 3 Statistical distribution of accuracy of daily wind power forecasting

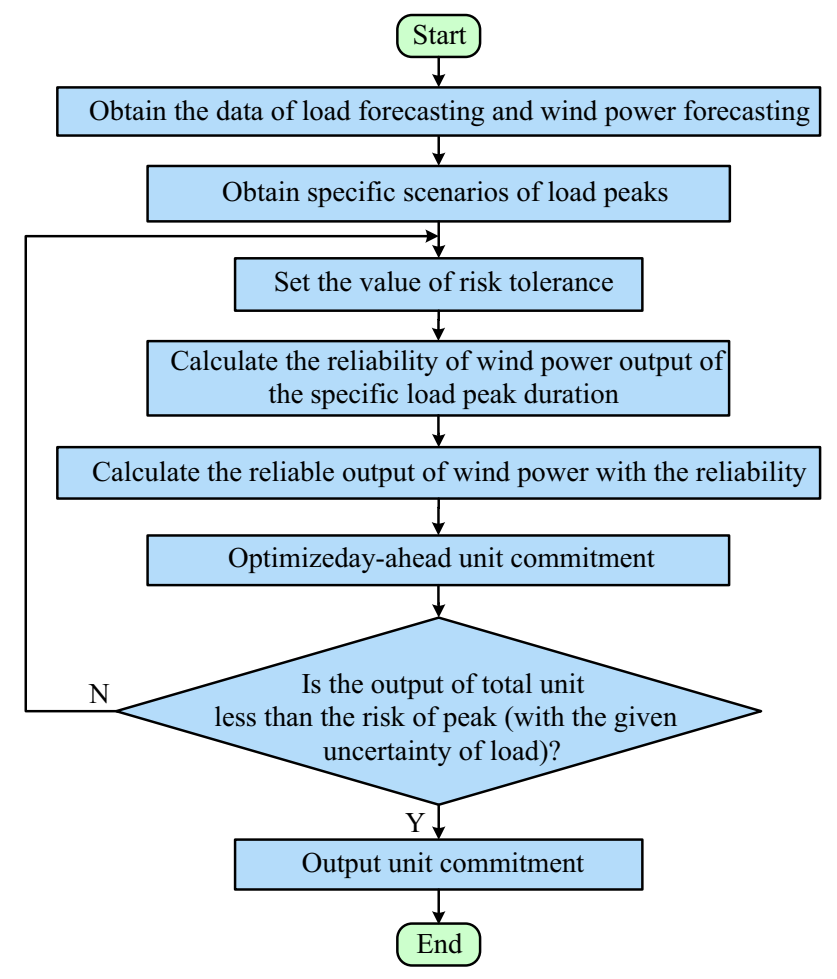

Fig. 4 Robust estimation flowchart of wind forecasting availability

wind power forecasting are: $r=0.12, P_{s}=0.08, v=0.1$, $c=0.92$. Set $e_{i}=10 \%$, and the distance function is $\chi^{2}$ according to divergence. The robust estimation algorithm of the availability of wind power forecasting in the next period of day-ahead dispatching of the local power grid is shown in Fig. 4, and is explained as follows:

1) According to durations of the load peaks of the power grid, the scenario mode is $d=(1,2,5,10,15,18,20$, $25,30,60)$, in terms of minutes. Based on the historical forecasting data, the probability vector with the wind power forecasting error $e_{i}=10 \%$ in each scenario is $\boldsymbol{p}_{0}=(0.80,0.82,0.85,0.81,0.93,0.85$, $0.91,0.93,0.91,0.93)^{\mathrm{T}}$.

2) Scenario samples with a capacity of $N=36,110,156$, $365,548,1095$ are obtained, corresponding to the load of one month, ten days, one week, three days, two days and one day, respectively. 100 samples are used to calculate the mean value.

3) As the samples are of different scales, based on the maximum likelihood estimation method, the samplebased probability in each peak load scene is $p_{i, N}(i=1$, $2, \ldots, m)$.

4) When the distance divergence function and confidence level $(1-K)$ are $95 \%$ and $99 \%$, respectively, and the fitness and risk tolerance $\beta$ are 0.03 and 0.09 , respectively, the solution of (15) is calculated to 
obtain the robust availability of the wind power forecasting.

Based on the forecasted value of wind power obtained from day-ahead dispatching, by the robust estimation model of wind power forecasting availability proposed in this study, the distribution of wind power forecasting availability with load peak duration of $15 \mathrm{~min}$ on the next day is calculated as shown in Fig. 5.

\subsection{Analysis of calculation of day-ahead dispatching based on wind power forecasting}

Based on the MATLAB simulation platform, this paper adopts the wind power forecasting credibility robust evaluation method when the load peak scenario appears. Taking for example a 39-node power system with $8000 \mathrm{MW}$ total capacities shown in Fig. 6, all the wind farms in the power grid are equivalent to one virtual power generation unit, and all other power sources are equivalent to a conventional thermal unit. The regulating capacity of the virtual wind power unit is set to $100 \%$, and the regulating capacity of the conventional thermal power unit is set to 50\%. In Fig. 6, "W" represents wind turbine.

The estimated curves of the day-ahead load and wind power forecasting are shown in Fig. 7. The grid-connected capacity of the wind farm takes $10 \%$ and $20 \%$ of the total capacity of the system, and the calculation time is $12.8 \mathrm{~s}$ and $14.2 \mathrm{~s}$, respectively, which is used to analyze the effectiveness of the model under different wind power conditions.

The model during the load peak of the power grid is $d=\{1,2,5,10,15,18,20,25,30,60\}$. According to the data of wind power forecasting, if the error of day-head

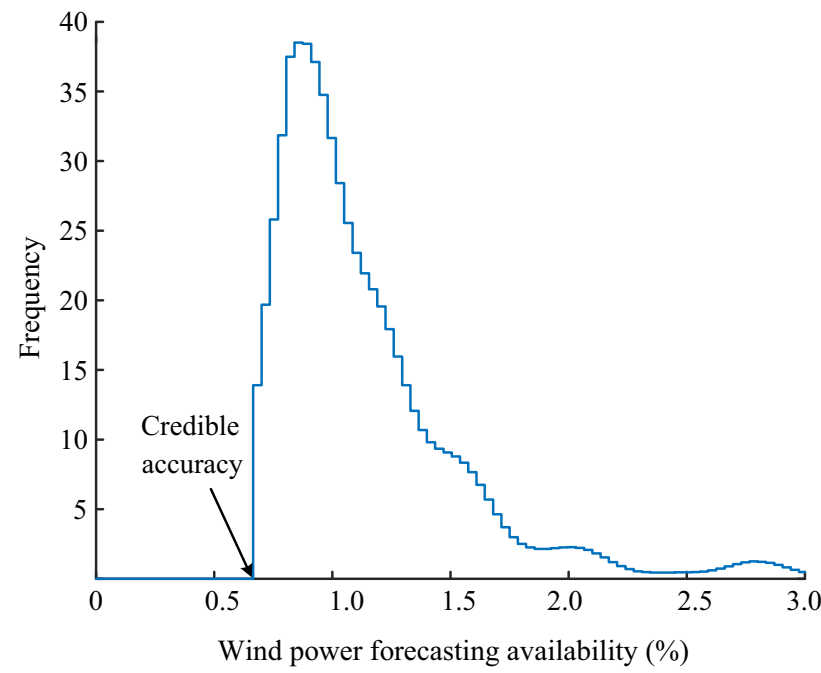

Fig. 5 Availability of wind power forecasting

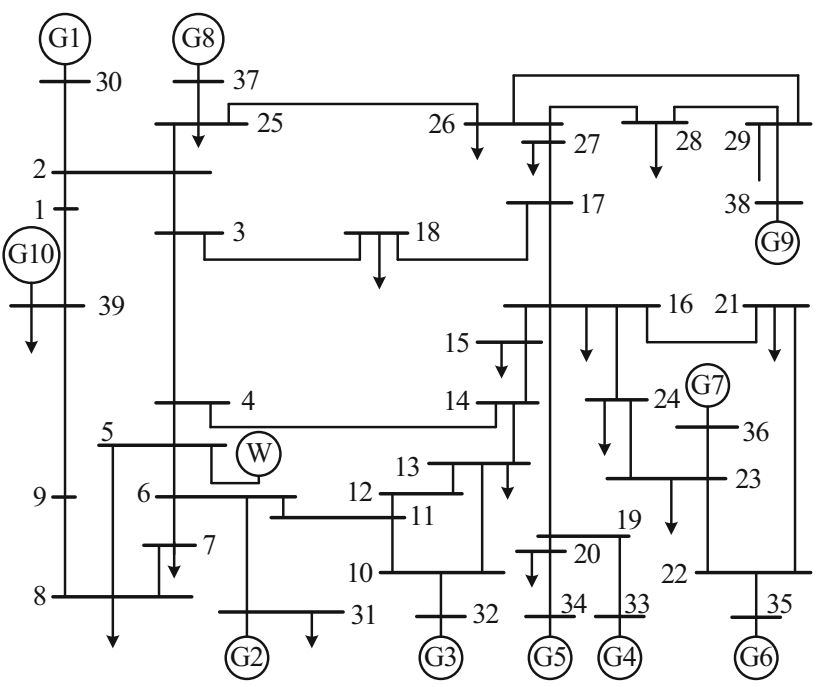

Fig. 6 Equivalent system diagram of power grid in one region

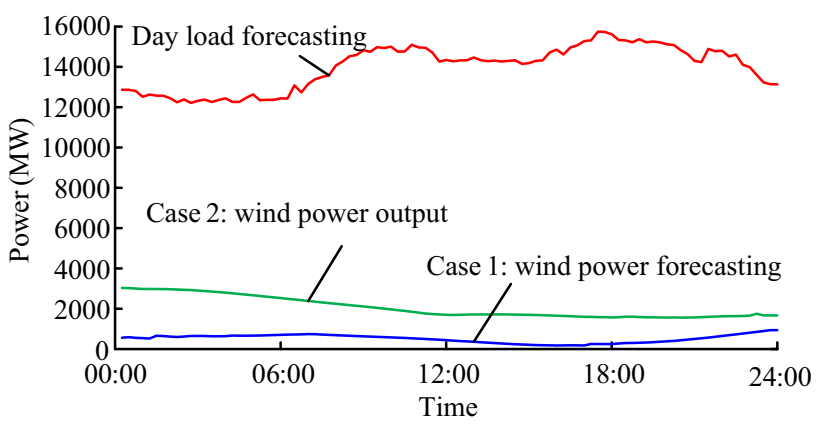

Fig. 7 Day-ahead load and wind power forecasting curves

wind power forecasting is $x_{i}=10 \%$, the probability of the day-ahead error under each period is $p_{0}=(0.80,0.82$, $0.85,0.81,0.93,0.85,0.91,0.93,0.91,0.93)^{\mathrm{T}}$.

According to the above results, by the robust estimation model of wind power forecasting availability, the day-head availability based on the scenarios of $\beta=0.03$ and $\beta=0.09$ is calculated, respectively. According to the calculated index of wind power availability, the maximum output power of the available virtual wind power units during the peak load of the power grid is calculated. The results are shown in Table 1.

According to the adjustable peak output value of the virtual wind power units in Table 1, the virtual units and the conventional thermal power units in the system are taken as the units with the same attribute for day-ahead dispatching. Unit commitment is shown in Table 2. Figure 8 shows the relationship between risk tolerance and availability.

The capacity of the connection of the power grids of the wind power field is $20 \%$ of the total capacity of the system, 
Table 1 Optimal confidence and condition risk value with different risk tolerances

\begin{tabular}{lll}
\hline Condition & $\begin{array}{l}\text { Optimal } \\
\text { availability }\end{array}$ & $\begin{array}{l}\text { Adjustable peak output of wind } \\
\text { power virtual unit (MW) }\end{array}$ \\
\hline $\begin{array}{l}\text { Wind power } \\
\text { ratio is } 10 \%, \\
\beta \text { is } 0.03\end{array}$ & 0.64 & 150 \\
$\begin{array}{l}\text { Wind power } \\
\text { ratio is } 10 \%,\end{array}$ & 0.68 & 163 \\
$\beta$ is 0.09 & \\
$\begin{array}{l}\text { Wind power ratio is } \\
\quad 0 \%, \beta \text { is } 0.03\end{array}$ & 0.61 & 286 \\
$\begin{array}{l}\text { Wind power } \\
\text { ratio is } 20 \%, \\
\beta \text { is } 0.09\end{array}$ & 0.65 & 312 \\
\end{tabular}

Table 2 Day-ahead unit commitment of dispatching

Risk tolerance $\beta$ Day-ahead unit commitment

$0.03 \quad$ W, G1, G2, G4, G6, G7, G8, G9, G11, G12, G15,

0.09

W, G1, G2, G3, G5, G7, G8, G10, G12, G14

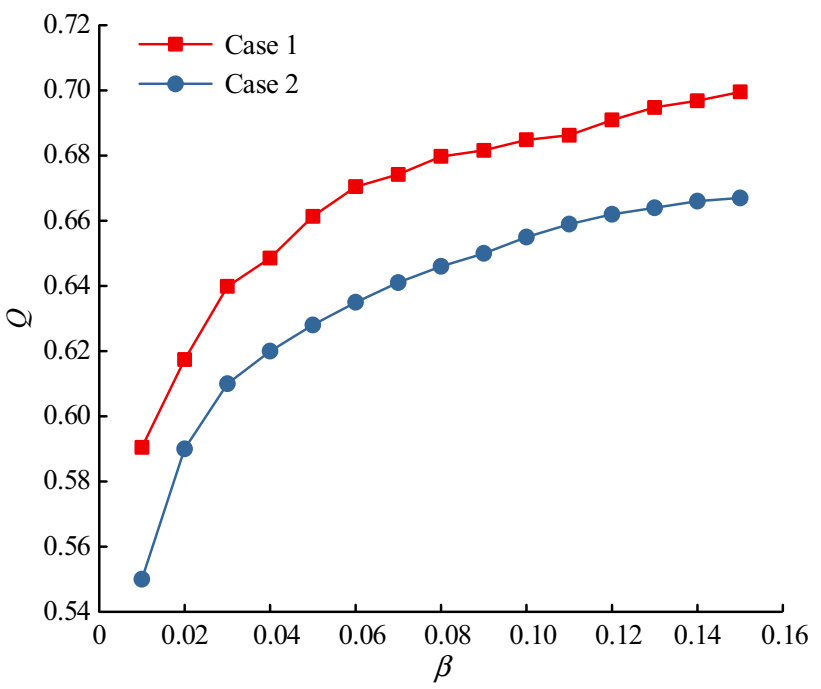

Fig. 8 Relationship between risk tolerance and availability

as we can see from Table 2. The product between the value of wind power forecasting and the index of credibility is equivalent to one unit, so the current number of units is reduced by 1 and 2 with the two risk tolerances, respectively. The number of running thermal power units is decreased.

The actual load of the power grid and the wind power output are assumed to be as shown in Fig. 9.

The simulation is proceeded with the two day-ahead unit commitment strategies of $\beta=0.03$ and $\beta=0.09$,

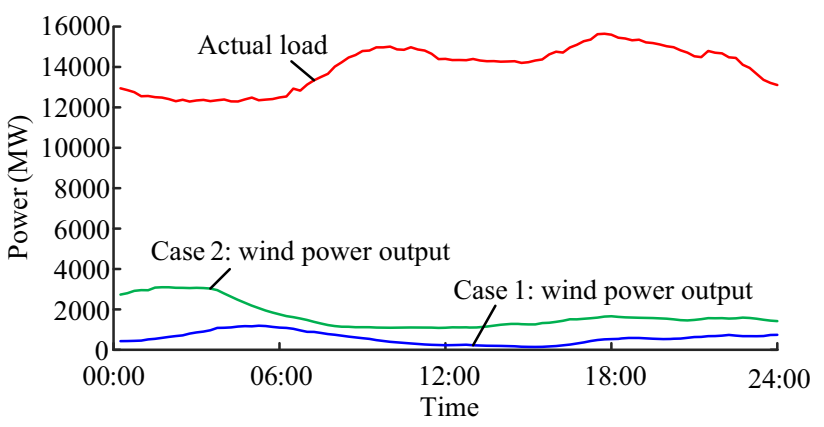

Fig. 9 Actual load and wind power output

respectively. The capacity of the connection of wind power accounts for $20 \%$ of the total capacity of the system. The unit commitment is equivalent to the availability of wind power forecasting, which is unconsidered. The counterpart is proceeded when the unit commitment policy of dayahead dispatching, as shown in Table 2, is considered.

Due to the uncertainty of wind power which is not involved in the units, the conventional day-ahead dispatching plan is hardly known to optimize the number of conventional thermal units and ensure the stability of the source power during the peak load period. Therefore, during the valley load period, the minimum output of the thermal unit occupies the room of wind power consumption, and curtailed wind is formed. As shown in Figs. 10 and 11, the adaption of the index of wind power forecasting makes the wind power consumption much better than that shown in Fig. 12, and hence the ratio of curtailed wind power of the grid power is reduced. The larger the risk tolerance is, the better wind power consumption will be. As a result, the reasonable index of risk tolerance can make the relationship controllable between wind power consumption and safe operation of power grid.

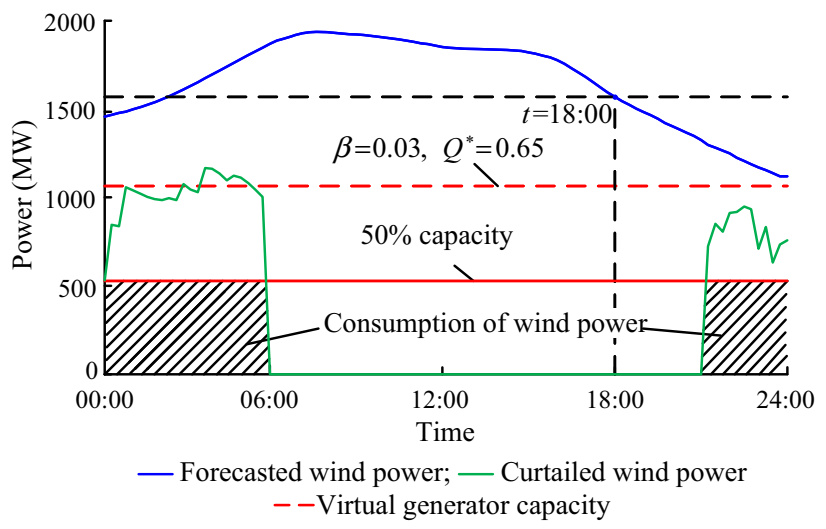

Fig. 10 Wind power consumption with day-ahead dispatching policy based on their availability when $\beta=0.03$ 


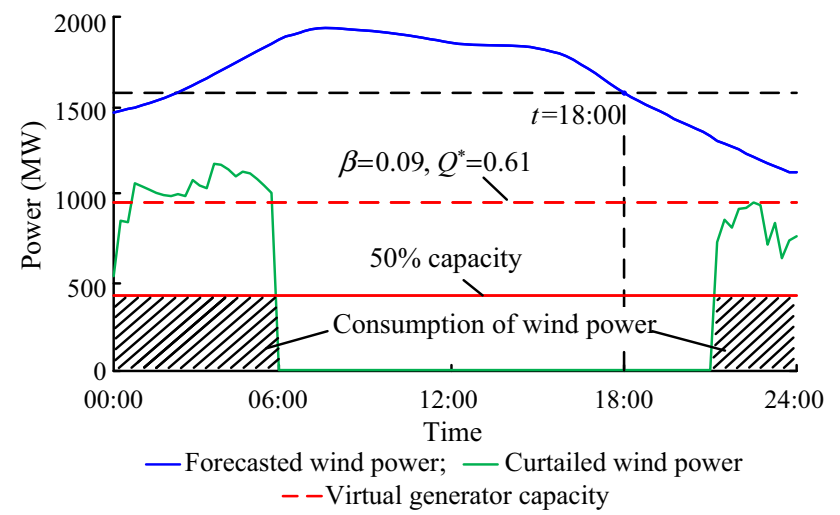

Fig. 11 Wind power consumption with day-ahead scheduling policy based on their availability when $\beta=0.09$

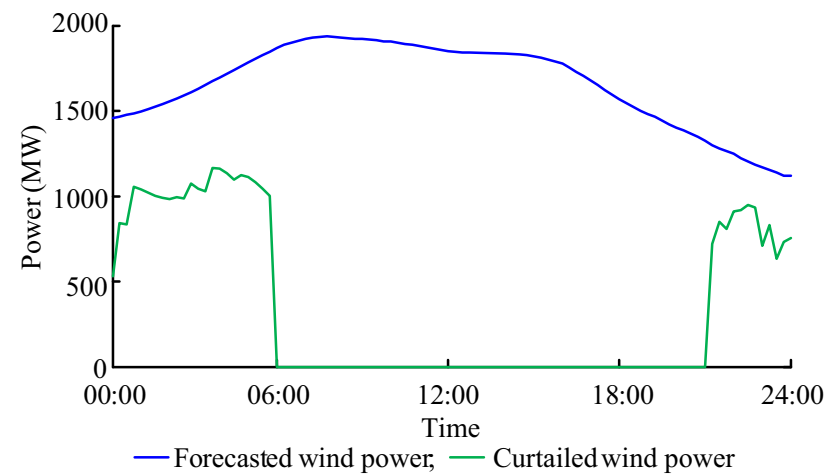

Fig. 12 Curtailed wind power with day-ahead scheduling policy of virtual unit ignoring their availability

\section{Conclusion}

In this paper, the availability index of wind power forecasting and the model of virtual wind power unit commitment with the minimum value of total wind power output during a selected period are proposed, based on the characteristics of historical data of the running power grid and the index of wind power forecasting evaluation.

Wind power forecasting credibility and its robust estimation model of probability distribution are proposed. The solution algorithm based on the robust plan is established.

The equivalent stimulating mode of 39-node power grid in 10 units is established. Based on the estimated model of the availability of wind power forecasting, the unit commitment is optimized. The simulation results show that the robust estimation of availability of wind power forecasting and the optimization of the unit commitment can effectively improve the wind power consumption of the power grid.

Acknowledgements This work was supported by the National Key Research and Development Program of China (No. 2017YFB0902100).
Open Access This article is distributed under the terms of the Creative Commons Attribution 4.0 International License (http:// creativecommons.org/licenses/by/4.0/), which permits unrestricted use, distribution, and reproduction in any medium, provided you give appropriate credit to the original author(s) and the source, provide a link to the Creative Commons license, and indicate if changes were made.

\section{References}

[1] Bruninx K, Delarue E (2014) A statistical description of the error on wind power forecasts for probabilistic reserve sizing. IEEE Trans Sustain Energy 5(3):995-1002

[2] Shil SK, Sadaoui S (2018) Meeting peak electricity demand through combinatorial reverse auctioning of renewable energy. J Mod Power Syst Clean Energy 6(1):73-84

[3] Liu F, Bie Z, Liu S (2017) Day-ahead optimal dispatch for wind integrated power system considering zonal reserve requirements. Appl Energy 188:399-408

[4] Teng Y, Sun P, Hui Q et al (2019) Multi-energy microgrid autonomous optimized operation control with electro-thermal hybrid storage. CSEE J Power Energy Syst. https://doi.org/10. 17775/cseejpes.2019.00220

[5] Zhang N, Kang C, Xia Q (2014) Modeling conditional forecast error for wind power in generation scheduling. IEEE Trans Power Syst 29(3):1316-1324

[6] Zhao L, Wei G, Zhi W et al (2018) A robust optimization method for energy management of CCHP microgrid. J Mod Power Syst Clean Energy 6(1):132-144

[7] Zarate-Minano R, Anghel M, Milano F (2013) Continuous wind speed models based on stochastic differential equations. Appl Energy 104:42-49

[8] Rajakovic NL, Shiljkut VM (2018) Long-term forecasting of annual peak load considering effects of demand-side programs. J Mod Power Syst Clean Energy 6(1):145-157

[9] Chang WY (2014) A literature review of wind forecasting methods. J Power Energy Eng 2(04):161-168

[10] Liu L, Ji T, Li M et al (2018) Short-term local prediction of wind speed and wind power based on singular spectrum analysis and locality-sensitive hashing. J Mod Power Syst Clean Energy 6(2):317-329

[11] Li H, Wang J, Lu H (2018) Research and application of a combined model based on variable weight for short term wind speed forecasting. Renew Energy 116:669-684

[12] Chen H, Li F, Wang Y (2018) Wind power forecasting based on outlier smooth transition autoregressive GARCH model. J Mod Power Syst Clean Energy 6(3):532-539

[13] Sedighi M, Moradzadeh M, Kukrer O et al (2018) Simultaneous optimization of electrical interconnection configuration and cable sizing in offshore wind farms. J Modern Power Syst Clean Energy 6(4):749-762

[14] Wang J, Du P, Niu T (2017) A novel hybrid system based on a new proposed algorithm-multi-objective whale optimization algorithm for wind speed forecasting. Appl Energy 208:344-360

[15] Zhang Z, Mei D, Jiang $\mathrm{H}$ et al (2018) Mode for reducing wind curtailment based on battery transportation. J Mod Power Syst Clean Energy 6(6):1158-1171

[16] Wang J, Niu T, Lu H et al (2018) An analysis-forecast system for uncertainty modeling of wind speed: a case study of largescale wind farms. Appl Energy 211:492-512 
[17] Yuan K, Zhang K, Zheng Y et al (2018) Irregular distribution of wind power prediction. J Mod Power Syst Clean Energy 6(6): $1172-1180$

[18] Chen F, Huang G, Fan Y et al (2017) A copula-based fuzzy chance-constrained programming model and its application to electric power generation systems planning. Appl Energy 187:291-309

[19] Jiang Y, Chen X, Yu K et al (2017) Short-term wind power forecasting using hybrid method based on enhanced boosting algorithm. J Mod Power Syst Clean Energy 5(1):126-133

[20] Quan H, Srinivasan D, Khambadkone AM et al (2015) A computational framework for uncertainty integration in stochastic unit commitment with intermittent renewable energy sources. Appl Energy 152:71-82

[21] Basit A, Hansen A, Sørensen P et al (2017) Real-time impact of power balancing on power system operation with large scale integration of wind power. J Mod Power Syst Clean Energy 5(2):202-210

[22] Wang Y, Zhang N, Kang C (2018) An efficient approach to power system uncertainty analysis with high-dimensional dependencies. IEEE Trans Power Syst 33(3):2984-2994

[23] Xie Y, Liu C, Wu Q (2017) Optimized dispatch of wind farms with power control capability for power system restoration. J Mod Power Syst Clean Energy 5(6):908-916

[24] Doostizadeh M, Aminifar F, Ghasemi H et al (2016) Energy and reserve scheduling under wind power uncertainty: an adjustable interval approach. IEEE Trans Smart Grid 7(6):2943-2952

Yun TENG received his Ph.D. degree in electrical engineering from Shenyang University of Technology, Shenyang, China, in 2009. He joined Shenyang University of Technology in 2010, where he is currently a Professor in electrical engineering. He is a standing director of the IEEE PES DC Distribution Network Technical Subcommittee. His research interests include multi-energy system dispatching automation and smart grid control theory.

Qian HUI received her M.Sc. degree in electrical engineering from Shenyang University of Technology, Shenyang, China, in 2017. She is currently pursuing her Ph.D. degree at the faculty of Electrical Engineering, Shenyang University of Technology. She joined the State Grid Liaoning Electric Power Research Institute Customer Service Center, China, in 2017. Her major research interests include new energy power systems and multi-energy coordinated dispatching.

Yan LI received his Ph.D. degree in electrical engineering from Shenyang University of Technology, Shenyang, China, in 2018. He joined the State Grid East Inner Mongolia Electric Power Supply Co. Ltd., China, in 2009, where he is currently an Electrical Engineer. His research interests include ultra-high voltage (UHV) substations, power transmission project construction, and power system simulation.

Ouyang LENG received her B.S. degree from North China Electric Power University, Beijing, China, in 2008. She joined the Economic and Technological Research Institute of the State Grid East Inner Mongolia Electric Power Co. Ltd., China, in 2016. Her major research directions include new energy power system and coordinated dispatching.

Zhe CHEN received his B.Eng. And M.Sc. degrees from Northeast China Institute of Electric Power Engineering, Changchun, China, and his Ph.D. degree from the University of Durham, Durham, U.K. $\mathrm{He}$ is a Full Professor with the Department of Energy Technology, Aalborg University, Aalborg, Denmark, where he is the leader of the Wind Power System Research program in the Department of Energy Technology. He is also the Danish Principle Investigator for Wind Energy of the Sino-Danish Centre for Education and Research. His research areas are power systems, power electronics and electric machines, and his main current research interests are wind energy and modern power systems. He has led many research projects and has authored or coauthored more than 400 publications in his technical field. Dr. Chen is a Fellow of the Institution of Engineering and Technology and a Chartered Engineer in the U.K. He is an editor of IEEE Transactions on Power Systems, and an associate editor of IEEE Transactions on Power Electronics and Journal of Modern Power Systems and Clean Energy. 